Boletín de la Sociedad Botánica de México 48: 19-22, 1988

DOI: $10.17129 /$ botsci.1342

Bol. Soc. Bot. México 48: 19-22 (1988)

\title{
Nuevos registros y nuevas combinaciones de gramíneas del Valle de México
}

\author{
YOLANDA HeRRERA A. ${ }^{1}$
}

\begin{abstract}
RESUMEN. Se citan por primera vez para el Valle de México 22 especies de gramíneas. De ellas, tres son aparentemente nuevos registros para México: Mublenbergia orophila Swallen (el tipo de Huehuetenango, Guatemala), Panicum miliaceum L. (maleza anual originaria de India) y Setaria faberi Herr. (maleza anual originaria de China). Otras tres especies probablemente se reportan por segunda vez para México: Mublenbergia bintonii Swallen, Mublenbergia seatoni Scribn. y Paspalum dilatatum Poiret. Se propone una nueva combinación para dos variedades de Andropogon sanguineus (Retz.) Merr.: Andropogon sanguineus (Retz.) Merr. var. brevipedicellatus (Beal) Herrera, basada en $A$. hirtiflorus (Nees) Kunth var. brevipedicellatus Beal y Andropogon sanguineus (Retz.) Merr. var. oligostachyus (Chapm.) Herrera, basada en $A$. oligostachyus Chapman.
\end{abstract}

ABSTRACT. Twenty two species of grasses from the Valley of Mexico are reported for the first time. Apparently, three of them are new records for Mexico: Mublenbergia orophila Swallen (Huehuetenango, Guatemala type), Panicum miliaceum L. (annual weed from India), and Setaria faberi Herr. (annual weed from China); and probably, three of them are reported for the second time: Mublenbergia bintonii Swallen, Mublenbergia seatoni Scribn., and Paspalum dilatatum Poiret. A new combination is proposed for two varieties of Andropogon sanguineus (Retz.) Merr.: Andropogon sanguineus (Retz.) Merr. var. brevipedicellatus (Beal) Herrera, based on A. birtiflorus (Nees) Kunth var. brevipedicellatus Beal, and andropogon sanguineus (Retz.) Merr. var. oligostachyus (Chapm.) Herrera, based on $A$. oligostachyus Chapm.

Como resultado del estudio de una parte de la familia Gramineae dentro del proyecto Flora y Vegetación del Valle de México, se encontraron 22 especies que no han sido mencionadas en la literatura como miembros de la flora del valle; algunas son especies de reciente introducción (caso típico de algunas malezas); otras no se conocían de la zona, ya sea por la poca profundidad con que se había tratado la familia Gramineae en anteriores estudios florísticos regionales, por errores en la identificación de ejempla-

${ }^{1}$ Laboratorio de Botánica Fanerogámica, Escuela Nacional de Ciencias Biológicas, Instituto Politécnico Nacional, 11410, México, D.F. Dirección actual: CIIDIR-IPN Unidad Durango, Hidalgo 120, 34890, Vicente Guerrero, Dgo.

Herrera-A Y. 1988. Nuevos registros y nuevas combinaciones de gramíneas del Valle de México. Boletín de la Sociedad Botánica de México 48: 19-22. 
res o por las diferencias en la delimitación del Valle de México en diversos trabajos (Reiche, 1926; Sánchez, 1969).

Mublenbergia hintonii Swallen

ESTADO DE MÉXICO. Municipio de Santa Ana Xilotzingo, 1-I-1974, Rzedowski 31662 (ENCB).

Especie escasa en el Valle, el tipo es: Hinton 3059, Temascaltepec, Estado de México. Aparentemente es el segundo reporte que se hace de esta especie, ya que se conocía solamente el de la localidad tipo.

Mublenbergia orophila Swallen

ESTADO DE MÉXICO. Municipio de Amecameca, 26-XI-1978, Rzedowski 36012 (ENCB); 23-XI-1980, Rzedowski 37111 (ENCB); Municipio de Tlalmanalco, XI-1980, Rzedowski 37190 (ENCB).

DISTRITO FEDERAL. Delegación de Cuajimalpa, 9-X-1977, Rzedowski 35362 (ENCB); 25-X-1983, Herrera 350 (ENCB).

Apartentemente ésta es la primera cita para México. El tipo es Steyermark 50309, Huehuetenango, Guatemala.

Mublenbergia polycaulis Scribn

ESTADO DE HIDALGO. Municipio de Epazoyucan, 10-IX-1972, Rzedowski 29321 (ENCB); 2-X-1982, Herrera 251 (ENCB).

Se conoce según McVaugh (1983: 251), del SW de EUA; Baja California, Sonora y Chihuahua a Jalisco y Guanajuato.

Mublenbergia seatoni Scribn

ESTADO DE HIDALGO. Municipio de Epazoyucan, 25-VIII-1984, Rzedowski 38485 (ENCB).

Aparentemente es el segundo reporte que se hace de esta especie, conocida sólo de la localidad tipo: Esperanza, Puebla, Seaton 320.

Panicum miliaceum L.

ESTADO DE MÉXICO: Municipio de Texcoco, 4-VIII-1966, Valdez y Aguirre sn (CHAPA, ENCB).

Especie originaria de la India, ampliamente cultivada en el viejo mundo como grano menor, introducida en varias partes de EUA; maleza anual recientemente introducida en el Valle de México. Excluida por McVaugh (1983) de la Nueva Galicia, probablemente ésta es la primera localidad comprobada para México.

Paspalum dilatatum Poiret

ESTADO DE MÉXICO. Municipio de Tepotzotlán, 7-VIII-1979, Rzedowski 36303 (ENCB); 2-VII-1972, Rzedowski 28998 (ENCB); 19-VIII-1971, Rzedowski 28573 (ENCB). Municipio de Cuautitlán, 7-VI-1980, González 32 (ENCB, MEXU); 7-VI-1980, González 24 (ENCB, MEXU). Municipio de Villa Nicolás Romero, 7-IX-1982, Ventura 4011 (ENCB).

Especie nativa en Sudamérica, desde Brasil a Argentina, introducida en el S de EUA 
como pasto de calidad forrajera. Citada en Baja California por Gould y Moran (1981: 114) y por Wiggins (1980: 947). Se ha citado de Jalisco, Díaz Pulido (1967: 189), pero fue excluida por McVaugh (1983) por no haberla encontrado en la Nueva Galicia. Aparentemente éste es el segundo registro comprobado para México.

Paspalum tinctum Chase

DISTRITO FEDERAL. Delegación Coyoacán, San Ángel, Gándara s.n. (MEXU).

Especie muy escasa, conocida por Chase (1929) de cuatro localidades, el tipo es: Hitchcock 7404, Irapuato. Se conoce de Jalisco, Michoacán, Guanajuato y con este registro del Valle de México.

Setaria faberi Herr.

ESTADO DE MÉXICO. Municipio de Cuautitlán, 7-VI-1980, Hernández 57 (CHAPA, ENCB).

Originaria de China, introducida en Estados Unidos (Rominger, 1962). En apariencia es la primera cita para México; con toda probabilidad introducida recientemente en el Valle.

Setaria verticillata (L.) Beauv.

ESTADO DE MÉXICO. Municipio de Cuautitlán, 8-X-1982, Hernández 332 (CHAPA, ENCB). Municipio de Texcoco, 21-X-1962, Rzedowski 16268 (ENCB).

DISTRITO FEDERAL. Delegación Gustavo A. Madero, 24-XII-1982, Hernández 456 (CHAPA, ENCB).

Maleza introducida en América proveniente de Europa, citada por Rominger (1962) de Massachusetts a Columbia Británica; SE de California; E de Virginia.

$\mathrm{Al}$ parecer especie de reciente introducción en el Valle de México. Citada por Beetle (1977), Wiggins (1980: 949) y Gould y Moran (1981: 120) de Baja California. Díaz Pulido (1967: 225) la cita para Jalisco, sin embargo, McVaugh (1983) la excluye de la Nueva Galıcia. Hitchcock (1931: 322) la menciona de Coahuila.

Otras 13 especies que se consideraron nuevos registros para el Valle de México, ya que no han sido reportadas específicamente para esta localidad, son las siguientes:

Andropogon gerardii Vitman Andropogon liebmannii Hack.

Digitaria ternata (Rich.) Stapf. Mublenbergia nigra Hitchc. Mublenbergia vaginata Swallen Panicum decolorans HBK. Panicum hallii Vasey
Panicum hians Ell.

Paspalum notatum Flugge

Paspalum plicatulum Michx.

Paspalum urvillei Steud.

Sorghastrum nutans (L.) Nash

Sorghum bicolor (L.) Moench.

\section{NUEVAS COMBINACIONES}

El género Andropogon ha sido dividido por algunos agrostólogos modernos en los géneros Andropogon, Botbriochloa, Dichanthium y Schizachyrium, con base en caracte- 
rísticas de la inflorescencia. En el estudio de la subfamilia Panicoideae (Gramineae) del Valle de México se reconoce solamente al género Andropogon, lo que hace necesario proponer combinaciones nuevas para dos variedades de Andropogon sanguineus (Retz.) Merr.

Andropogon sanguineus (Retz.) Merr. var. brevipedicellatus (Beal) Herrera, comb. nov. Andropogon birtiflorus (Nees) Kunth var. brevipedicellatus (Beal), Grasses of North America, 2:44. 1896. TIPO: MÉXICO (Chihuahua), Pringle 383 (Us).

Andropogon sanguineus (Retz.) Merr. var. oligostachyus (Chapm.) Herrera, comb. nov. Andropogon oligostachyus (Champ.) Flora Southeastern U.S. 581. 1860. TIPO: FLORIDA (Middle), Chapman s.n.

\section{LITERATURA CITADA}

Beetle, A.A., 1977. Noteworthy grasses from Mexico V. Phytologia 37(4): 317-407.

Chase, A., 1929. The North American species of Paspalum. Contr. U.S. Natl. Herb. 28(1): 310.

Díaz Pulido, C.I., 1967. Contribución al conocimiento de las gramíneas de Jalisco. Tesis, Facultad de Ciencias, UnAM. México. 251 pp.

Gould, F. W. y R. Morán., 1981. The grasses of Baja California, México. San Diego Society of Natural History Memoir 12. 140 pp.

Hitchcock, A.S., 1931. (Poales) Poaceae (pars). North Amer. Fl. 17(4): 289-354.

Mcvaugh, R., 1983. Flora Novo-Galiciana. Vol. 14 Gramineae. University of Michigan. 1032 pp.

Reiche, C., 1926. Flora excursoria en el Valle Central de México. Talleres Gráficos de la Nación, México, D.F. 303 pp.

Rominger, J.M., 1962. Taxonomy of Setaria (Gramineae) in North America. Illinois Biol. Monogr. 29:127.

SÁnchez, O., 1969. La Flora del Valle de México. Ed. Herrero, México, D.F. 519 pp.

Wiggins, I.L., 1980. Flora of Baja California. Stanford University Press. 1025 pp. 